Int. Journal of Math. Analysis, Vol. 7, 2013, no. 1, 43 - 49

\title{
Poincare Bifurcation of a Kind of Hamiltonian System under Polynomial Perturbation ${ }^{1}$
}

\author{
Yongkang Zhang ${ }^{2}$
}

LMIB and School of Mathematics and Systems Science

Beihang University, Beijing, 100191, P.R. China

\section{Cuiping Li}

LMIB and School of Mathematics and Systems Science

Beihang University, Beijing, 100191, P.R. China

\begin{abstract}
It was proved that the sharp upper bound of the number of zeros of Abelian integrals and the number of limit cycles bifurcated from Poincare bifurcation were $B(n)$. An explicit $B(n)$ is derived for the number of zeros of Abelian integrals $I(h)=\oint_{\Gamma(h)} f(x, y) d y-g(x, y) d x$ on the open interval $(0, \infty)$, where $\Gamma(h)$ is an oval lying on the algebraic curve $H(x, y)=x^{2 a} / A+y^{2 b} / B=h, f(x, y), g(x, y)$ are polynomials of $x$ and $y$, and $\max \{\operatorname{deg} f(x, y), \operatorname{deg} g(x, y)\}=n$. Assume $I(h)$ not vanish identically, $c=\operatorname{gcd}(a, b), \lambda=\max \{a / c, b / c\}$, then $B(n)=\frac{1}{2}\left[\frac{n-1}{2}\right]\left(\left[\frac{n-1}{2}\right]+3\right)$ for $n \leq 2 \lambda, B(n)=\lambda\left[\frac{n-1}{2}\right]-\frac{1}{2}(\lambda-1)(\lambda-2)$ for $n \geq 2 \lambda+1$.
\end{abstract}

Subject Classification: 34C07, 34C08

Keywords: Abelian integrals, Hamiltonian system, Poincare bifurcation

\section{Introduction and statement of the main re- sults}

Consider a real polynomial $H(x, y)$ of degree $d+1$ in the plane. A closed connected component of a level curve $H=h$ is denoted by $\Gamma(h)$ and called an oval of $H$. Let $\Sigma=\left(h_{1}, h_{2}\right) \subset \mathbb{R}^{1}$ be a maximal interval of existence of $\Gamma(h)$.

\footnotetext{
${ }^{1}$ Supported by Key Program of NNSF No. 61134005.

${ }^{2}$ Author for correspondence: changyongkang@ss.buaa.edu.cn.
} 
Let $\omega=-f(x, y) d y+g(x, y) d x$ be a real 1-form with polynomial coefficients of degree at most $n$. Define the complete Abelian integral

$$
I(h)=\oint_{\Gamma(h)} \omega, \quad h \in \Sigma .
$$

Any small perturbation corresponds to a polynomial vector field of degree $n$ reads

$$
d H+\epsilon \omega=0 .
$$

Here, $\epsilon$ is a small parameter. The general problem of estimating the number of limit cycles in a polynomial unfolding (2) of a polynomial system having a center, is known as the weakened Hilbert's 16th problem, initially posed by Arnold[1]. The problem reduces to the study of the first order approximation term $I(h)$ of the corresponding displacement map

$$
d(h, \epsilon)=\epsilon I(h)+O\left(\epsilon^{2}\right) .
$$

Namely, the zeros of $I(h)$ in the interval $\Sigma$ where the level sets $H=h$ from a continuous family of ovals (period annuli), correspond to the limit cycles emerging from this period annulus for $\epsilon$ small (i.e. generated by Poincare bifurcation).

The weakened Hilbert's problem has been solved only in its original form and in the quadratic case $n=2$. It is known in this case that the function $I(h)$ can have at most two zeros. The general problem is still open even in the case $n=2$. The general results about this problem was achieved in $[6,11]$, where the existence of an upper bound $Z(d, n)$, the number of zeros of the integral (1), was proved, but its explicit expression is not solved except for some special cases. Petrov[10] achieved $Z(2, n) \leq n-1$ for $H(x, y)=y^{2}+x^{3}-x$. Li \& Zhang[7] obtained $M_{2}(h)$ has at most $2(n-1)$ zeros for $n$ even and at most $2 n-3$ zeros for $n$ odd when $M_{1}(h) \equiv 0$ for $H(x, y)=y^{2}+x^{3}-x$. Gavrilov[2] gave $Z(2, n) \leq[(2 / 3)(n-1)]$ for $H(x, y)=(1 / 2)\left(x^{2}+y^{2}\right)-(1 / 3) x^{3}+x y^{2}([p]$ denotes the entire part of $p)$. Horozov \& Iliev[3] concluded $Z(2, n) \leq 5 n+15$ for the Abelian integrals corresponding to cubic Hamiltonians. Zhao \& Zhang[12] stated $Z(3, n) \leq 7 n+5$ for $H(x, y)=(1 / 2) y^{2}+U(x)$, where $\operatorname{deg} U(x)=4$. Iliev[4] achieved $M_{k}(h)$ has at most $k(n-1)$ zeros, counting the multiplicity, where $M_{k}(h)$ is the first Melnikov function in (3) not vanishing identically for $H(x, y)=(1 / 2)\left(x^{2}+y^{2}\right)-(1 / 3) x^{3}$. And further references therein $[5,8,9,13]$.

In this paper, we consider one kind of Hamiltonian with symmetry under polynomial perturbation

$$
\left\{\begin{array}{l}
\dot{x}=\frac{\partial H(x, y)}{\partial y}+\epsilon f(x, y) \\
\dot{y}=-\frac{\partial H(x, y)}{\partial x}+\epsilon g(x, y)
\end{array},\right.
$$


where $H(x, y)=x^{2 a} / A+y^{2 b} / B, A, B$ are positive real numbers, and $a, b$ are positive integers, $f(x, y)$ and $g(x, y)$ are polynomials in $x$ and $y$ with real coefficients, and $\max \{\operatorname{deg} f(x, y), \operatorname{deg} g(x, y)\}=n, \epsilon$ as given above in (2). In the present paper, we investigate the limit cycles generated from the Poincare bifurcation of the above system and obtain the following main result. Additionally, in the following gcd (resp. lcm) is short for greatest common divisor (resp. least common multiple).

Theorem 1.1 Assume $I(h)$ is not vanish identically, $c=\operatorname{gcd}(a, b), \lambda=$ $\max \{a / c, b / c\}$. Then for $(4), B(n)=\frac{1}{2}\left[\frac{n-1}{2}\right]\left(\left[\frac{n-1}{2}\right]+3\right)$ for $n \leq 2 \lambda, B(n)=$ $\lambda\left[\frac{n-1}{2}\right]-\frac{1}{2}(\lambda-1)(\lambda-2)$ for $n \geq 2 \lambda+1$. $B(n)$ is the sharp upper bound of the number of zeros of Abelian integrals $I(h)$ and the number of limit cycles bifurcated from Poincare bifurcation of (4).

The rest of this paper is organized as follows. In Sec. 2, $I(h)$ can be expressed as a polynomial of new variable $u$, which is

$$
A(u)=\sum_{i+j \leq\left[\frac{n-1}{2}\right]} a_{i j} u^{i \alpha+j \beta} .
$$

In Sec. 3, we can derive the number of terms of $A(u)$ and obtain the number of zeros of $A(u)$, using these results, we prove our main Theorem 1.1.

\section{Preliminary}

We derive the following preliminary results which will be used later on. The Abelian integral for system (4) is the following

$$
\begin{aligned}
I(h) & =\oint_{\Gamma_{h}} g(x, y) d x-f(x, y) d y \\
& =\iint_{\operatorname{Int} \Gamma_{h}} \sum_{i+j \leq n-1} c_{i j} x^{i} y^{j} d x d y \\
& \left.=\iint_{\operatorname{Int} \Gamma_{h}} \sum_{i+j \leq\left[\frac{n-1}{2}\right]} c_{2 i, 2 j} x^{2 i} y^{2 j} d x d y \quad \text { (by symmetry }\right) \\
& =\oint_{\Gamma_{h}} \sum_{i+j \leq\left[\frac{n-1}{2}\right]} \frac{c_{2 i, 2 j}}{2 j+1} x^{2 i} y^{2 j+1} d x \\
& =4 \int_{\substack{\Gamma_{h} \\
y \geq 0}} \sum_{i+j \leq\left[\frac{n-1}{2}\right]} \frac{c_{2 i, 2 j}}{2 j+1} x^{2 i} y^{2 j+1} d x .
\end{aligned}
$$


Let

$$
\left\{\begin{array}{l}
x=x(h, \theta)=A^{\frac{1}{2 a}} h^{\frac{1}{2 a}}(\cos \theta)^{\frac{1}{a}} \\
y=y(h, \theta)=B^{\frac{1}{2 b}} h^{\frac{1}{2 b}}(\sin \theta)^{\frac{1}{b}}
\end{array} \quad, \quad \theta \in\left[0, \frac{\pi}{2}\right]\right.
$$

then

$$
\begin{aligned}
I(h)= & 4 \int_{\frac{\pi}{2}}^{0} \sum_{i+j \leq\left[\frac{n-1}{2}\right]} \frac{c_{2 i, 2 j}}{a(2 j+1)}\left(A^{\frac{1}{2 a}} h^{\frac{1}{2 a}}(\cos \theta)^{\frac{1}{a}}\right)^{2 i}\left(B^{\frac{1}{2 b}} h^{\frac{1}{2 b}}(\sin \theta)^{\frac{1}{b}}\right)^{2 j+1} \\
& \times A^{\frac{1}{2 a}} h^{\frac{1}{2 a}}(\cos \theta)^{\frac{1}{a}-1}(-\sin \theta) d \theta \\
= & \sum_{i+j \leq\left[\frac{n-1}{2}\right]} a_{i j} h^{\frac{2 i+1}{2 a}+\frac{2 j+1}{2 b}}
\end{aligned}
$$

where

$$
a_{i j}=\frac{2 c_{2 i, 2 j}}{a(2 j+1)} A^{\frac{2 i+1}{2 a}} B^{\frac{2 j+1}{2 b}} \mathscr{B}\left(\frac{2 i+1}{2 a}, \frac{2 j+2 b+1}{2 b}\right),
$$

and $\mathscr{B}(p, q)$ is a Beta function, $\mathscr{B}(p, q)=2 \int_{0}^{\frac{\pi}{2}}(\cos \theta)^{2 p-1}(\sin \theta)^{2 q-1} d \theta \neq 0$. Assume $\operatorname{gcd}(a, b)=c$, then there exist positive integers $\alpha, \beta$ suffice to $a=\alpha c$, $b=\beta c$, and $\operatorname{gcd}(\alpha, \beta)=1$, also $\lambda=\max \{a / c, b / c\}=\max \{\alpha, \beta\}, \operatorname{lcm}(a, b)=$ $\alpha \beta c$. Take $u=h^{\frac{1}{\alpha \beta c}}$, then $u \in(0, \infty)$ and

$$
\begin{aligned}
I(h) & =\sum_{i+j \leq\left[\frac{n-1}{2}\right]} a_{i j} h^{\frac{(2 i+1) \alpha+(2 j+1) \beta}{2 \alpha \beta c}} \\
& =u^{\frac{\alpha+\beta}{2}} \sum_{i+j \leq\left[\frac{n-1}{2}\right]} a_{i j} u^{i \alpha+j \beta} .
\end{aligned}
$$

Denote $A(u)=\sum_{i+j \leq\left[\frac{n-1}{2}\right]} a_{i j} u^{i \alpha+j \beta}, u \in(0, \infty)$. Next section we study the property of polynomial $A(u)$ in $(0, \infty)$.

\section{Proof of Theorem 1.1}

Lemma 3.1 Denote the set $X:=\{p \gamma+q \delta \mid p, q \in \mathbb{N}, p+q \leq K, \gamma, \delta \in$ $\left.\mathbb{N}^{+}, \operatorname{gcd}(\gamma, \delta)=1, \gamma \geq \delta\right\}$, then $|X|=\frac{1}{2}(K+1)(K+2)$ for $K \leq \gamma-1$, $|X|=\gamma K+1-\frac{1}{2}(\gamma-1)(\gamma-2)$ for $K \geq \gamma$, where $|X|$ denote the number of elements in the set $X$.

Proof Obviously it holds for $\gamma=1$. When $\gamma>1$, denote $A_{m}=\{l \gamma+m \mid$ $l \in \mathbb{Z}\}, B_{m}=\{l \gamma+m \delta \mid l \in \mathbb{Z}\}$. Since $\operatorname{gcd}(\gamma, \delta)=1$, all the divisor from $\gamma$ by $0, \delta, 2 \delta, \cdots,(\gamma-1) \delta$ is different each other. Hence, $B_{0}, B_{1}, \cdots, B_{\gamma-1}$ is a rearrangement of $A_{0}, A_{1}, \cdots, A_{\gamma-1}$. By $A_{i} \cap A_{j}=\emptyset(i \neq j)$ and $\bigcup_{m=0}^{\gamma-1} A_{m}=\mathbb{Z}$, 
we obtain $B_{i} \bigcap B_{j}=\emptyset(i \neq j), \bigcup_{m=0}^{\gamma-1} B_{m}=\mathbb{Z}, X=\bigcup_{m=0}^{\gamma-1}\left(B_{m} \bigcap X\right)$, and $|X|=\sum_{m=0}^{\gamma-1}\left|B_{m} \bigcap X\right|$.

Furthermore,

$$
\begin{gathered}
B_{0} \cap X=\{p \gamma \mid 0 \leq p \leq K\} \Rightarrow\left|B_{0} \cap X\right|=K+1, \\
B_{1} \cap X=\{p \gamma+\delta \mid 0 \leq p \leq K-1\} \Rightarrow\left|B_{1} \cap X\right|=K, \\
B_{2} \cap X=\{p \gamma+2 \delta \mid 0 \leq p \leq K-2\} \Rightarrow\left|B_{2} \cap X\right|=\max \{0, K-1\}, \\
\cdots, \\
B_{\gamma-1} \cap X=\{p \gamma+(\gamma-1) \delta \mid 0 \leq p \leq K-(\gamma-1)\} \Rightarrow\left|B_{\gamma-1} \cap X\right|=\max \{0, K-\gamma+2\} .
\end{gathered}
$$

Hence,

$$
|X|=\sum_{m=0}^{\gamma-1} \max \{0, K-m+1\}
$$

then,

$$
|X|=(K+1)+K+\cdots+2+1=\frac{1}{2}(K+1)(K+2)
$$

for $K \leq \gamma-1$, and

$$
|X|=(K+1)+K+\cdots+(K-\gamma+2)=\gamma K+1-\frac{1}{2}(\gamma-1)(\gamma-2)
$$

for $K \geq \gamma$.

Lemma 3.2 Denote $F(x)=\sum_{k=0}^{m} C_{k} x^{a_{k}}, 0=a_{0}<a_{1}<\cdots<a_{m}$, then the isolated zeros of $F(x)$ in $(0, \infty)$ is less than $m$, and there exist the real numbers $C_{i}, i=0, \cdots, m$, let $F(x)$ has exactly $m$ simple zeros in $(0, \infty)$.

Proof Without any loss of generality, assume $\sum_{i=0}^{m} C_{i}^{2} \neq 0$.

Firstly, we get the isolated zeros $F(x)$ in $(0, \infty)$ is less than $m$ by the induction. Suppose it holds for $m=l-1$, then for $m=l$,

$$
F^{\prime}(x)=\sum_{k=0}^{l} C_{k} a_{k} x^{a_{k}-1}=x^{a_{1}-1}\left(C_{1} a_{1}+C_{2} a_{2} x^{a_{2}-a_{1}}+\cdots+C_{l} a_{l} x^{a_{l}-a_{1}}\right)
$$

where $C_{1} a_{1}+C_{2} a_{2} x^{a_{2}-a_{1}}+\cdots+C_{l} a_{l} x^{a_{l}-a_{1}}$ has $l-1$ zeros at most in $(0, \infty)$. From Rolle Theorem, $F(x)$ has $l$ zeros at most in $(0, \infty)$.

Secondly, one elaborate there exist real numbers $C_{i}, i=0, \cdots, m$, let $F(x)$ has exactly $m$ simple zeros in $(0, \infty)$. One can put $C_{0}=1$, suppose $F(x)=\sum_{k=0}^{m} C_{k} x^{a_{k}}=0$ has $m$ positive roots $1,2, \cdots, 2^{m-1}$, then $\sum_{k=0}^{m} C_{k}=$ 
$0, \sum_{k=0}^{m} C_{k} 2^{a_{k}}=0, \cdots, \sum_{k=0}^{m} C_{k}\left(2^{m-1}\right)^{a_{k}}=0$. Consider a set of $m$ linear equations in $m$ unknowns $C_{1}, C_{2}, \cdots, C_{m}$, and

$$
\sum_{k=0}^{m} C_{k}=-1, \sum_{k=0}^{m} C_{k} 2^{a_{k}}=-1, \cdots, \sum_{k=0}^{m} C_{k}\left(2^{m-1}\right)^{a_{k}}=-1 .
$$

The determinant of coefficient matrix $D$ of above linear system is,

$$
\begin{aligned}
\operatorname{det} D & =\left|\begin{array}{cccc}
1 & 1 & \cdots & 1 \\
2^{a_{0}} & 2^{a_{1}} & \cdots & 2^{a_{m}} \\
\cdots & \cdots & \cdots & \cdots \\
\left(2^{m-1}\right)^{a_{0}} & \left(2^{m-1}\right)^{a_{1}} & \cdots & \left(2^{m-1}\right)^{a_{m}}
\end{array}\right| \\
& =\left|\begin{array}{cccc}
1 & 1 & \cdots & 1 \\
2^{a_{0}} & 2^{a_{1}} & \cdots & 2^{a_{m}} \\
\left(2^{a_{0}}\right)^{2} & \left(2^{a_{1}}\right)^{2} & \cdots & \left(2^{a_{m}}\right)^{2} \\
\cdots & \cdots & \cdots & \cdots \\
\left(2^{a_{0}}\right)^{m-1} & \left(2^{a_{1}}\right)^{m-1} & \cdots & \left(2^{a_{m}}\right)^{m-1}
\end{array}\right| \\
& =\prod_{0 \leq i<j \leq m}\left(2^{a_{j}}-2^{a_{i}}\right) \neq 0
\end{aligned}
$$

Hence, the $m$ th linear equation exist unique real solution $C_{1}, C_{2}, \cdots, C_{m}$, so as to $F(x)$ at least has $m$ zeros in $(0, \infty)$.

Combine the two parts, $F(x)$ has exactly $m$ simple zeros in $(0, \infty)$.

Proof[Proof of Theorem 1.1] From Lemma 3.1, for $n \leq 2 \lambda, K=\left[\frac{n-1}{2}\right] \leq$ $\lambda-1$, polynomial $A(u)$ has $\frac{1}{2}\left(\left[\frac{n-1}{2}\right]+1\right)\left(\left[\frac{n-2}{2}\right]+2\right)$ terms, by Lemma 2 , the zeros of $A(u)$ in $(0, \infty)$ is less than $B(n)=\frac{1}{2}\left(\left[\frac{n-1}{2}\right]+1\right)\left(\left[\frac{n-2}{2}\right]+2\right)-1=$ $\frac{1}{2}\left[\frac{n-1}{2}\right]\left(\left[\frac{n-2}{2}\right]+3\right)$. Similarly, for $n \geq 2 \lambda+1, K=\left[\frac{n-1}{2}\right] \geq \lambda$, polynomial $A(u)$ has $\lambda\left[\frac{n-1}{2}\right]+1-\frac{1}{2}(\lambda-1)(\lambda-2)$ terms, the positive zeros of $A(u)$ is less than $B(n)=\lambda\left[\frac{n-1}{2}\right]+1-\frac{1}{2}(\lambda-1)(\lambda-2)-1=\lambda\left[\frac{n-1}{2}\right]-\frac{1}{2}(\lambda-1)(\lambda-2)$.

From Lemma 3.2, we can get the sharp upper bound $B(n)$ of positive zeros by construct the real numbers $C_{i}, i=0, \cdots, m(i . e$. construct polynomials $f(x, y)$ and $g(x, y))$. The number of zeros of Abelian integrals $I(h)$ equals the positive zeros of polynomial $A(u)$, which the sharp upper bound both is $B(n)$.

From above discussion, $I(h)$ has exactly $B(n)$ simple zeros in $(0, \infty)$, that is, system (4) have $B(n)$ hyperbolic limit cycles in a neighborhood of the periodic orbits $\Gamma_{h}$. Therefore, the sharp bound of the limit cycles generated by Poincare bifurcation of system (4) also is $B(n)$.

\section{References}

[1] V. I. Arnold, Loss of stability of self-oscillation close to resonance and 
versal deformation of equivariant vector fields, Funct. Anal. Appl. 11, (1977) 1-10.

[2] L. Gavrilov, Nonoscillation of elliptic integrals related to cubic polynomials with symmetry of order three, Bull. Lond. Math. Soc. 30, (1998) 267-273.

[3] E. Horozov, \& I. D. Iliev, Linear estimate for the number of zeros of Abelian integrals with cubic Hamiltonians, Nonlinearity 11, (1998) 15211537.

[4] I. D. Iliev, On the limit cycles available from polynomial perturbations of the Bogdanov-Takens Hamiltonian, Israel J. Math. 115, (2000) 269-284.

[5] J. Jiang, \& M. Han, Melnikov function and limit cycle bifurcation from a nilpotent center, Bull. Sci. Math. 132, (2008) 182-193.

[6] A. G. Khovansky, Real analytic manifolds with finiteness properties and complex Abelian integrals, Funct. Anal. Appl. 18, (1984) 119-128.

[7] B. Li, \& Z. Zhang, A note of a G. S. Petrov's result about the weakened 16th Hilbert problem, J. Math. Anal. Appl. 190, (1995) 489-516.

[8] C. Li, et al. Polynomial systems: A lower bound for the weakened 16th Hilbert problem, Extracta Math. 16, (2001) 441-447.

[9] J. Llibre, \& X. Zhang, On the number of limit cycles for some perburbed Hamiltonian systems, Dyn. Contin. Discrete Impuls. Syst. Ser. A Math. Anal. 8, (2001) 161-168.

[10] G. S. Petrov, Elliptic integrals and their non-oscillatorness,Funct. Anal. Appl. 20, (1986) 46-49.

[11] A. N. Varchenko, Estimate of the number of zeros of an Abelian integral depending on a parameter and limit cycles, Funct. Anal. Appl. 18, (1984) 98-108.

[12] Y. Zhao, \& Z. Zhang, Linear estimate of the number of zeros of Abelian integrals for a kind of quartic Hamiltonian, J. Diff. Eqns. 155, (1999) 73-88.

[13] Y. Zhao, \& Z. Zhang, Abelian integrals and period functions for quasihomogeneous Hamiltonian vector fields, Applied Math. Letters 18, (2005) 563-569.

Received: August, 2012 